\title{
Klinische Pharmakologen und Toxikologen in der Schweiz
}

Mitsuko Kondo-Oestreicher

Korrespondenz:

Dr. med.

Mitsuko Kondo-Oestreicher

HUG

Pharmacologie et

toxicologie cliniques

Rue Gabrielle-Perret-Gentil 4

1211 Genève 14

Tel. 0223729004

mitsuko.kondo-oestreicher@ hcuge.ch

www.clinpharm.ch

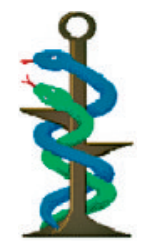

Ärzte, Apotheker und andere in klinischer Pharmakologie und Toxikologie spezialisierte Akademiker sind in Krankenhäusern, staatlichen Einrichtungen, Gesundheitsbehörden, privatwirtschaftlichen Unternehmen oder als Niedergelassene tätig. Ärzte mit Spezialisierung auf klinische Pharmakologie haben mindestens 3 Jahre Weiterbildung in einem Grundlagengebiet wie Innere Medizin, Anästhesie oder Pädiatrie sowie weitere drei Jahre fachspezifische Weiterbildung absolviert [1] . Apotheker und Akademiker anderer Fächer müssen ausser ihrer grundlegenden Qualifikation mindestens vier Jahre Weiterbildung in klinischer Pharmakologie vorweisen.

\section{Klinische Pharmakologie: Verbesserung der Patientenversorgung}

Ähnlich wie in anderen Ländern strebt die klinische Pharmakologie auch in der Schweiz das Ziel an, dass Arzneimittel wirksam, rational, angemessen, sicher und kontrolliert angewendet werden («ERASM use»: effective, rational, adapted, safe and monitored). Dies geschieht durch verschiedene Aktivitäten wie zum Beispiel Erprobung und Einführung neuer Therapieoptionen. Klinische Pharmakologen haben hierfür verschiedene konzeptionelle Instrumente entwickelt, beispielsweise die klinische Pharmakokinetik, Pharmakodynamik, Pharmakogenetik, Pharmakoepidemiologie, Pharmakovigilanz, Pharmakometrie, Pharmakoökonomie und soziale Pharmakologie. Klinische Pharmakologen sind häufig in klinischen Gremien vertreten und an Angeboten auf nationaler Ebene beteiligt. Typische Aufgaben auf lokaler Ebene umfassen etwa die Leitung der Medikamentenkommissionen der Krankenhäuser, Entwicklung und Pflege von Arzneimittellisten, Beurteilung neuer Präparate, Erstellung und Überwachung von Verschreibungsleitlinien, Prüfung von Vorfällen in der medikamentösen Therapie sowie die Förderung der evidenzbasierten Therapie [2].

\section{Klinische Toxikologie: Beratung und Behandlung bei Vergiftungen} Die wichtigsten Tätigkeitsfelder der klinischen Toxikologie sind die Beratung (im Rahmen des Schweizerischen Toxikologischen Informationszentrums und der regionalen Universitätszentren für klinische Pharmakologie), die Behandlung von Vergiftungen und Überdosierungen bei Menschen sowie die Auswertung von Daten zu versehentlicher und absichtlich herbeigeführter Exposition gegenüber Arzneimitteln, Chemikalien und Pflanzen (Toxikovigilanz). Auf allen Ebenen arbeiten klinische Pharmakologen und Toxikologen eng mit weiteren Gesundheitsberufen zusammen. Im Bereich der Diagnostik und Therapie beispielsweise besteht reger Kontakt zum verschreibenden bzw. behandelnden Arzt und zu den Patienten; im Bereich der Arzneimittelbereitstellung zum lokalen niedergelassenen bzw. Krankenhausapotheker und zum Pflegepersonal; beim Drug-Monitoring zu Chemikern, Biochemikern und Biologen; bei der Überwachung von Arzneimittelnebenwirkungen (Pharmakovigilanz und Toxikovigilanz) zu regionalen, nationalen und internationalen Behörden; in der klinischen Forschung schliesslich zu Prüfärzten, Studienmonitoren, Ethikkommissionen und lokalen Gesundheitsbehörden. Ausserdem gehört es zu ihren Aufgaben im Dienste der Allgemeinheit, wissenschaftlich fundierte, unparteiische und nützliche Informationen über Arzneimittel und Chemikalien zu verbreiten.

\section{Die Schweizerische Gesellschaft}

für Klinische Pharmakologie und Toxikologie

Die klinischen Pharmakologen und Toxikologen sind eng in die akademische Lehre und Weiterbildung eingebunden, für die sie Lehrinstrumente und -ressourcen für optimale Arzneimittelverschreibung entwickeln.

Dies ist eines der wichtigen Felder, in denen die SGKPT ihre Mitglieder unterstützt. Weitere bedeutende Aktivitäten der SGKPT sind die klinische Forschung, Vermittlung der guten klinischen Praxis, die Translationsforschung, die Weiterverbreitung evidenzbasierter Therapieleitlinien und die Bereitschaft, in Arzneimittelfragen als Experten zur Verfügung zu stehen.

\section{Literatur}

1 www.fmh.ch/de/data/pdf/klinische_pharmakologie_ version_internet_d.pdf.

2 Consultant physicians working with patients. $4^{\text {th }}$ edition. London: Royal College of Physicians; 2008 . 